\title{
Part 3: Mobility
}

\author{
Travelers with Disabilities: Challenges and Assis- \\ tive Technologies \\ Alireza Darvishy* \\ Institute of Information Technology, Zurich Univer- \\ sity of Applied Sciences, Steinberggasse 13, 8400 Win- \\ terthur, Switzerland
}

Background: This paper describes the preliminary results an ongoing project in the area of accessible tourism, which is currently being run by different universities, industrial partners and selected hotels. The first goal of the project is to systematically identify the barriers that travelers with disabilities encounter today when going on holidays. Another goal of this project is to build so-called Hotel-Living-Labs (HLLs). These are hotel rooms that are generally accessible and which are equipped with assistive technologies as well as assistive services. The HLLs serve as a test bed where assistive technologies and services can be evaluated in a realistic holiday environment. The last goal is to iteratively build an overall service for travelers with disabilities who wish to enjoy accessible holidays in a customer-centered way, together with the different customer groups with disabilities and their accompanying persons.

Method: Firstly, a literature review of potential barriers for travelers with disabilities and elderly travelers was performed, and assistive technologies/services were proposed for overcoming these barriers. These identified potential barriers are currently being verified in a series of ongoing interviews, which are being conducted with 25 individuals, separated into four categories: persons with vision impairments, persons with hearing impairments, wheelchair users, and elderly persons. Each interview lasts 1 hour. The findings from the interviews will be compared with the findings of the literature review. The results will then be used to develop a number of fully accessible "hotel living lab" rooms, where the travelers' experiences will be evaluated via a questionnaire.

Key results: The initial literature review revealed that, apart from facilities or services not being accessible themselves, many of the identified potential barriers are related to information offered by missing, inaccessible, outdated, inaccurate or inadequate information offered to the guests by the providers of assistive technologies/services before and during the holiday stay.

Conclusion: Accessible Tourism is an important goal to achieve in order to allow travelers with disabilities and elderly travelers to enjoy their holidays to the same degree as anyone else. Because the preliminary results of this project have indicated that many of the existing barriers occur because important information is missing, inaccurate, or inaccessible at different steps of the holiday journey, the project will focus on applying digital assistive technologies that allow this information to be provided in an accessible manner.

Keywords: Accessible tourism, interviews, assistive technologies

*Corresponding author. E-mail: alireza.darvishy@ zhaw.ch

\section{People Moving in a Smart City}

Laura Burzagli $^{\mathrm{a}, *}$ and Pier Luigi Emiliani ${ }^{\mathrm{a}}$

anstitute of Applied Phisics "Nello Carrara”, CNR, Via Madonna del Piano 10, 50019 Sesto Fiorentino, Florence, Italy

Background: In Italy, several projects for the deployment of ICT infrastructures to support the smart city concept are active. One of the main components is the control of the vehicles traffic. However, the needs of pedestrians should be considered, aiming to guide their navigation in the city and to offer support if they have problems. The system should be inclusive and personalized, including: (i) people without specific requirements (e.g. with context-based instructions and allowing requests in free format); (ii) fragile people as old people offering additional support (e.g. telling them where they are, if they are lost, and guiding them home); (iii) people with activity limitations, with supports based on their specific limitation (e.g. giving accessible information about the environment). 
Method: An innovative structured procedure will be used, based on the identification of the functionalities necessary to support the activities listed in Chapter 4 of the WHO ICF classification, d450 Walking, d460 Moving around in different locations, d470 Using transportation, $\mathrm{d} 475$ Driving, their organization in services and the integration in social networks. This approach has been tested in a system developed in our laboratory, which addresses Chp. 6 Domestic life, in particular feeding and Chp. 7, Interpersonal interactions and relationship. It allows the choice of a recipe (with control of health problems), the automatic management of the pantry and of the shopping list, the stepby-step assistance in the preparation of dishes. When people do not have the necessary abilities, AT is used when suitable (e.g. for the interface adaptation) or special support is offered (for example simplification of the procedures for people with cognitive difficulties). Useful comments have been collected during the evaluation though an (adapted) Pluralistic Usability Walkthrough (PUW). Then, the knowledge acquired in the implementation of a second system dealing with a psychological problem of people, who live alone, i.e. solitude, will integrated introducing elements of "intelligent" support for adaptation to people and situations. This system identifies the situation of people from their behavior and trough a learning component supports them with suggestions (not decisions). from the system itself and/or from the social network - An evaluation of the system is under preparation in a sheltered condominium in Florence.

Key results: The main result is the conceptual verification of the structured design procedure of support systems, which starts from ICF and is based on the support by technology, by connected people, and by artificial intelligent components to learn from their real use and to adapt them to the individual person. This implies the functionalities of a navigation system, the cooperation of people (in the social environment or in vicinity of the moving person), and intelligence in the system, to collect information about the situation and reactions of moving people and their social environment, reasoning on these data to devise personalized suggestions.

Conclusion: The above-cited results appear good starting points to be generalized to a more complex environment, together with the identification of the impact on assistive technology suitable for its use in a mobile and public environment.

Keywords: Services, ICF, Mobility.

*Corresponding author. E-mail: 1.burzagli@ifac.cnr.it
Challenges in Indoor Navigation and Accessibility Vikas Upadhyay $^{\mathrm{a}}$, Neha Jadhav ${ }^{\mathrm{b}, *}$, Tigmanshu Bhatnagar $^{\text {c }}$, Pranay ${ }^{\mathrm{d}}$, P.V.M Rao ${ }^{\mathrm{e}}$ and M. Balakrishnan ${ }^{\mathrm{f}}$ $\mathrm{a}, \mathrm{b}, \mathrm{f}$ School of Information Technology, IIT Delhi, India ${ }^{\mathrm{c}, \mathrm{d}, \mathrm{e}}$ Department of Design, IIT Delhi, India

Background: Independent mobility is a primary concern for inclusion of visually impaired (VI) people. Indoor mobility is challenging because of information unavailability, structural complexity, poor representation and lack of orientation and mobility training. Adoption of new technology including security concerns creates more complications in independent accessibility. Some of the critical indoor facilities that demand accessibility includes hospitals, and transport terminal. This work is an effort to create a more reliable technological solution for accessible indoor navigation for VI. Current research like NavCog and Soundscape for supporting indoor and outdoor navigation focuses on improving localization accuracy and audio-based information delivery but have challenges like reliability and cognitive load. Empirically we found, indoor navigation requires contextual localization accuracy and hierarchical information delivery for better non-visual cognitive mapping while most of blind navigation methods do not include this. Our key motivation here is to investigate how an indoor navigation representation scheme works for VI and how technology could play a role in inclusive navigation interface design.

Method: Our approach is based on creation of information representation scheme influenced from sighted navigational cues (like decision point, progressive disclosure and information reinforcement) from indoor. We have done a preliminary survey to understand indoor navigation cues for sighted. The indoor representation scheme for navigation using regular signage (already tested in a hospital) helps us to understand and annotate building maps with navigation and safety related information (entry/exit, doors) and services (lifts, stairs, ramp, drinking water and washrooms) required by all the users. We include additional annotations like floor surface change (e.g. small steps or ramps) and protrusion in the corridors (e.g. fire extinguishers) to enhance accessibility and to generate a rich annotated map with additional meta-data. One important step we are examining to get scalability to other public buildings is the possibility of extracting required annotations from videos. We use beacon and user input-based course localization and audio-based feedback using smart-phone. We are now in the process of conducting 
a study with VI users to test our system. It is expected that such representation scheme and associated interventions can contribute to open standards for indoor navigation and accessibility.

Key results: We have some key findings from our limited trials in a public hospital in New Delhi, India. The emphasis has been on an inclusive solution due to a diverse demography of users and affordability that involves effective utilization of existing infrastructure to make the solution cost-effective and scaleable. Our study has shown that providing right information at right place can be very effective in indoor spaces. Trials have shown that prior information about upcoming landmarks within indoor spaces is helpful. Progressive disclosure of information and timely reinforcement can help in reducing cognitive load. Ongoing research states that non-visual cognitive mapping of environment can enhance retention using such cues.

Conclusion: This paper deals with problem of indoor navigation for VI. We found right information at right time is a key to reliable indoor navigation. Our limited trials suggest that an information representation scheme and contextual localization can handle the diversity in user group and should be included in open standards for indoor navigation and accessibility.

Keywords: Accessibility, Indoor navigation, Mobility, Visually impaired, Cognitive map, Localization.

*Corresponding author. E-mail: neha.manik.jadhav@ cse.iitd.ac.in

\section{An Affordable Concept to Produce Mobility De- vices in Low Resource Settings \\ Valdis Krumins $^{\mathrm{a}}{ }^{*}$, Luc de Witte ${ }^{\mathrm{b}}$, Sapna Behar ${ }^{\mathrm{c}}$ and Varshapriya Radhakrishnan ${ }^{\mathrm{c}}$ \\ ${ }^{a}$ Advanced Manufacturing Research Centre, Univer- sity of Sheffield, Wallis Way, Catcliffe, Rotherham S60 $5 T Z, U K$ \\ ${ }^{\mathrm{b}}$ Centre for Assistive Technology and Connected Healthcare, University of Sheffield, 217 Portobello, Sheffield S1 4DP, UK \\ ${ }^{\mathrm{c}}$ Icarus Nova Discovery Pvt Ltd, No: 7 Rogers Road, Richard's Town, Bangalore 560 005, India}

Background: In many low resource settings even the most basic assistive devices are not available, and if available, they are often not affordable for the people who need them. Therefore the World Health Organisation (WHO) has identified the development of affordable high quality assistive devices as a global research priority. In this presentation we present the results of a project in which we looked at the possibility of producing frequently used assistive devices in more efficient ways, and make them more suitable for the specific context of low resource settings. We focused on mobility aids as listed in the WHO Priority Assistive Products List. With an international team of experts in manufacturing technologies, product design, assistive technology provision and rehabilitation professionals in India and the UK we developed an innovative concept for locally producing mobility devices.

Method: The project consisted of three phases. In the first phase we studied the most urgent needs in two areas in India, one in the city of Delhi and one in a rural area near Bangalore, using a survey method. In a second phase we made a technical analysis of the WHO Priority Assistive Products List from the perspective of more efficient production methods, and organized a multidisciplinary and international workshop to discuss the link between needs on the one hand and the possibilities of more efficient production methods on the other. This led to three promising possibilities: Adaptation and modification of existing AT solutions, End-user involved modular assistive devices and Assistive Technology App. In the third phase one of these was concretely developed into an innovative production concept by a team of product designers in the UK and India.

Key results: The result is a concept for producing three types of mobility devices (walking stick, crutches and walker frame). During the presentation this concept will be demonstrated and the approach followed to reach this result discussed. The concept is easy to produce at a low cost, devices can be generated locally 'on the spot' and individualized for the user, parts can be re-used and repair is very easy using basic hand tools. The personalisation has been incorporated in the assembly process by cutting the tubular components to a specific length for the particular end user. By removing the assembly process from the centralised manufacturer of the modular components and introducing it to local distribution centres the assembly costs are converted into jobs for the local community. A field trial in rural India with the new concept is being planned.

Conclusion: This project shows that it is possible to develop innovative concepts and methods that enable the production and assemblage of affordable and high quality assistive products. Multidisciplinary and international collaboration proved to be essential to achieve this. The resulting concept for mobility devices has the potential to greatly contribute to better availability in low resource settings. 
Keywords: mobility, affordable, crutch, walker, produced locally.

*Corresponding author. E-mail: v.krumins@ sheffield. ac.uk

User Acceptance of Augmented Reality Glasses for Hand Exoskeleton Control

Tobias Ableitner $^{\mathrm{a}, *}$, Sandra Metzl ${ }^{\mathrm{a}}$, Surjo Soekadar ${ }^{\mathrm{b}, \mathrm{c}}$, Andreas Schilling ${ }^{\mathrm{d}}$, Christophe Strobbe ${ }^{\mathrm{a}}$ and Gottfried Zimmermann $^{\mathrm{a}}$

${ }^{a}$ Responsive Media Experience Research Group Stuttgart Media University, Nobelstraße 10, Stuttgart 70569, Germany

${ }^{\mathrm{b}}$ Clinical Neurotechnology Laboratory, Neuroscience Research Center (NWFZ) \& Department of Psychiatry and Psychotherapy, Charité - University Medicine Berlin, Charitéplatz 1, Berlin 10117, Germany

${ }^{\mathrm{c}}$ Applied Neurotechnology Laboratory, University Hospital Tübingen, Calwerstraße 14, Tübingen 72076, Germany

${ }^{\mathrm{d}}$ WSI / GRIS, University of Tübingen, Sand 14, Tübingen 72076, Germany

Background: Every year about 260,000 people in Germany suffer a stroke, of which one third retain severe motor deficits. Particularly, loss of hand function leads to a considerable loss of autonomy and quality of life. We expect an actuated hand exoskeleton to provide them with daily life assistance and restore their hand function. However, hand exoskeleton control poses a considerable challenge for human-machine interaction. We follow up on Markovic et al. (2014) who have achieved promising results in hand prosthesis control using AR glasses.

Method: In a preliminary study, we investigated which AR glasses are suitable and acceptable by the users. In general, there are two types of AR glasses: (1) Large glasses with a real AR experience (e.g. Microsoft HoloLens); (2) Small glasses that resemble a head-up display (e.g. Epson BT-300, Google Glass). In order to investigate which type has higher user acceptance for controlling a hand exoskeleton, we implemented a prototypical assistance system without hand exoskeleton for the HoloLens and the BT-300. We evaluated them with 6 healthy test persons (age: $\mathrm{M}=25.50 \mathrm{SD}=$ 7.71). They indicated on a Likert scale $(1=$ not at all, $5=$ definitely) whether they would use the respective AR glasses to control an assistance system for the following application contexts: $(\mathrm{C} 1)$ private, $(\mathrm{C} 2)$ public, (C3) rehabilitation environment. We also asked them how long they would comfortably use them in general.
Based on the results of the preliminary study, the main study was a questionnaire focusing exclusively on small AR glasses. We asked 15 patients (10 stroke patients, 5 tetraplegics, age: $\mathrm{M}=54.33 \mathrm{SD}=13.45$ ) about their experiences with AR glasses and their theoretical acceptance for controlling a hand exoskeleton. All 15 patients had motor or sensory limitations in at least one hand. 8 of them could not grasp any objects with at least one hand and would benefit from a hand exoskeleton. Again, we assessed the user acceptance on a Likert scale. We also collected additional qualitative data.

Key results: The preliminary study showed that the subjects would wear the BT-300 $(\mathrm{M}=48.10 \mathrm{~min}$ SD $=42.27 \mathrm{~min}$ ) significantly longer than the HoloLens $(M=20.50 \mathrm{~min} S D=15.00 \mathrm{~min})$. Furthermore, the BT-300 (C1: $\mathrm{M}=4.00 \mathrm{SD}=1.09, \mathrm{C} 2: \mathrm{M}=3.33 \mathrm{SD}$ $=1.63$ and $\mathrm{C} 3: \mathrm{M}=4.00 \mathrm{SD}=1.55)$ has a higher user acceptance than the HoloLens $(\mathrm{C} 1: \mathrm{M}=3.33 \mathrm{SD}=$ $1.03, \mathrm{C} 2: \mathrm{M}=2.00 \mathrm{SD}=0.89, \mathrm{C} 3: \mathrm{M}=3.17 \mathrm{SD}=$ 2.04).

In the main study, the 15 patients showed a high user acceptance $(\mathrm{C} 1: \mathrm{M}=4.28 \mathrm{SD}=1.44, \mathrm{C} 2: \mathrm{M}=2.93$ $\mathrm{SD}=1.75, \mathrm{C} 3: \mathrm{M}=5.00 \mathrm{SD}=0.00$ ) for small $\mathrm{AR}$ glasses to control the hand exoskeleton. However, 5 patients indicated that they would not wear AR glasses for at least one application context.

Conclusion: This study shows that small AR glasses have a high user acceptance for assistive system control in private and clinical environments. This brings about novel interaction possibilities with assistive systems. However, the results also show that - to date many assistive system users do not want to wear AR glasses in public. Therefore, it is necessary that assistive systems such as hand exoskeletons can be operated by other input and output devices, aside from glasses. Keywords: hand exoskeleton, augmented reality, assistive technology, user acceptance.

*Corresponding author. E-mail: tobias.ableitner@ googlemail.com

Accuracy evaluation of an add-on acquisition system of operation log with inertial measurement units for a mobility scooter

Shunsuke Kurokawa ${ }^{a, b}$, Jun Suzurikawa ${ }^{\text {, } *}$, Takenobu Inoue $^{\mathrm{b}}$ and Kazunori Hase ${ }^{\mathrm{a}}$

${ }^{a}$ Department of Mechanical Systems Engineering, Tokyo Metropolitan University, 1-1, Minamiosawa, Hachioji-shi, Tokyo 192-0397, Japan

${ }^{\mathrm{b}}$ Department of Assistive Devices, National Reha- 
bilitation Center for Persons with Disabilities, 4-1, Namiki, Tokorozawa-shi, Saitama 359-8555, Japan

Background: In recent years, a mobility scooter spreads in society as a mobility aid for elderly people and plays a considerable role in maintaining the quality of life. Despite its convenience, severe accidents have happened with increasing number of users. To reduce accidents, appropriate measures of driving in daily use will be a possible solution. Therefore we developed a simple and easy-to-install acquisition system of operation $\log$ for detection of dangerous driving in daily use. The feature of this system is capability to quantify and record operations of the mobility scooter by only a few inertial measurement units (IMUs). The coordinate transformation of gravitational acceleration measured by IMU attached on the steering tiller can estimate two operation variables, i.e., the angles of the steering handlebar and the accelerator lever. This study aims to evaluate the estimation accuracy of these two variables and to optimize the combination of IMUs on the scooter body and the tiller.

Method: [Estimation method] The acquisition system is composed of two IMUs mounted on the body and the accelerator lever of the mobility scooter and the data logger on the front basket. The relation between the two operation variables and the gravitational accelerations measured by the IMU on the lever can be described by the coordinate transformation with rotation matrices. Solving this equation enables to convert the measurement data into the two operation variables. The IMU on the scooter body is utilized to compensate for the body inclination.

[Accuracy evaluation] The estimation accuracy of the proposed system was examined by changing the numbers of IMUs and axes (i.e. acceleration and/or angular velocity) used for calculation. The true values of the angles were measured by wire displacement sensors. The test course included flat and sloped surfaces. The similarity between the estimated and the true values was evaluated using the root mean square error (RMSE) and the correlation coefficient (CC).

Key results: The number of IMUs used for calculation mainly influenced the estimation accuracy with the presence of terrain inclination. The inclination compensation by the IMU on the scooter body was effective in the sloped surface with the inclination of over 7 degree whereas the inclination-induced estimation error was ignorable with flat surfaces. The use of angular velocities measured by IMUs was another factor impacting on the estimation accuracy. In some cases, the angle of the accelerator lever was estimated with better accuracy when the angular velocity was complementally used with acceleration. The estimation accuracy of the steering angle, on the other hand, was the best when estimated solely by acceleration measured with two IMUs. RMSE and CC with this estimation condition were 3.18 degree and 0.99 , respectively.

Conclusion: The estimation accuracy of the operation variables of the mobility scooter were compared with different methods using acceleration and angular velocity. Consequently, we concluded that the combination use of 3-axis acceleration values measured by two IMUs is suitable for monitoring driving behavior in daily use.

Keywords: IMU, Power wheelchair, Skills evaluation, Lifelog.

*Corresponding author. E-mail: suzurikawa-jun@ rehab.go.jp

\section{A Semantic Decision Support System to foster Re- turn to Work of Novice Wheelchair Users}

Daniele Spoladore ${ }^{\mathrm{a}, *}$, Sara Arlati $^{\mathrm{a}}$, Margherita Fossati $^{b}$, Luca Negri ${ }^{b}$, Marco Sacco ${ }^{a}$ and Mario Cocchi $^{\mathrm{b}}$

${ }^{a}$ Institute of Intelligent Industrial Technologies and Systems for Advanced Manufacturing, (STIIMA), National Research Council of Italy (CNR), 23900 Lecco, Italy

${ }^{\mathrm{b}}$ Scientific Institute, IRCCS E. Medea, 23842 Bosisio Parini, Lecco, Italy

Background: This work presents a Decision Support System (DSS) to foster return to work (RTW) of novice wheelchair users (NWU). The DSS is developed within an ongoing research project financed by the Italian National Institute for Insurance against Accidents at Work (INAIL). Following accidents at work, many NWUs decide not to come back to work, thus relying on the National Institute of Welfare for disability pensions; however, long-term unemployment could have a negative impact on NWU's mental and physical health. Within this context, the DSS aims at easing the process of RTW for NWUs by supporting therapists and vocational personnel with technological means in discriminating the still suitable jobs for each specific user.

Method: The DSS leverages semantic representations of the knowledge related to jobs and NWUs' functional capacity evaluation; for the first representation, O*NET (Occupational Information Network) vocabulary (the cross sections "Skills" and "Abilities", 
which are common to all the professions mapped in $\mathrm{O} * \mathrm{NET}$ ) was converted into an ontology. For the second, four comprehensive Core Sets from the International Classification of Functioning, Disability and Health (ICF) - corresponding to the three primary conditions that force a person on a wheelchair: Spinal Cord Injury, Traumatic Brain Injury, Stroke, plus the Vocational Rehabilitation Core Set to address work reintegration - were modelled into an ontology to describe the functioning of NWUs. Each O*NET skill and ability describing a job was then translated into ICF, via a consensus forum involving two psychologists and two biomedical engineers. Using ICF qualifiers and O*NET importance scores for each "Skills" and "Abilities", the DSS leverages a set of rules to automatically match a NWU's functional capacity evaluation, performed with ICF, to a list of jobs he/she can still perform.

Key results: Up to date, the DSS can model any NWU's condition with ICF and match him/her with a list of possible jobs; the DSS includes 10 professions (4 clericals, 4 physicals, 2 middle-management), but its structure allows to easily add more jobs. The outcomes deriving from the reasoning processes of the DSS can highlight the skills and abilities in which the NWU may face some difficulties, thus helping the vocational personnel and therapists in deciding whether to suggest the NWU to return to a specific job, perhaps with the support of some facilitators.

Conclusion: The presented DSS, leveraging semantic web technologies and ICF, provides a common language for clinical and vocational personnel and allows the involvement of non-clinical personnel (employers), with the aim of fostering work reintegration of NWUs and enhancing their quality of life, thus decreasing the impact of work accident consequences in terms of private and social costs. In the following months, the presented DSS will be tested for technology acceptance and usability (using respectively TAM 3 and SUS scales) by INAIL personnel.

Keywords: wheelchair user, return to work, decision support system, semantic web, ontology.

*Corresponding author. E-mail: daniele.spoladore@ stiima.cnr.it

\section{Wheelchair Users' Experiences with and Need of Activity Trackers \\ Tina Helle ${ }^{\mathrm{a}, *}$ and Kirstine Rosenbeck Gøeg ${ }^{\mathrm{b}}$ \\ ${ }^{a}$ University College of Norther Denmark, TIBS, Selma \\ Lagerlфfs Vej 2, 9220 Aalborg Øst, Denmark}

\section{${ }^{\mathrm{b}}$ Rosenbeck Informatics, Oluf Borchs vej 39, 9000 Aal-} borg, Denmark

Background: The use of different kinds of activity trackers, e.g. step counters, has become popular to monitor one's physical activity level. A typical goal for users is to evaluate whether public health recommendations stated by health authorities are met. Given a sedentary lifestyle, wheelchair users are particularly challenged when it comes to the performance of physical activity. However, activity trackers on the market are not applicable for wheelchair users. Obviously, steps are not a valid measure, but research suggest that other measures such as heart rate, wheel rotation and arm acceleration also have limitations. Yet, wheelchair users still use activity trackers, and their experiences are key to developing better technology.

The aim was to investigate: What are the wheelchair users' experiences of using current technological devices for activity tracking and, what are their requirements for future valid and meaningful activity tracking?

Method: A focus group interview was conducted, involving $\mathrm{N}=7$ wheelchair users; one woman and seven men in the age of 24-49. Participants were sampled thorough a wheelchair users basketball club. The focus group lasted for one hour. A semi-structured interview guide was developed to guide the interview. A content analysis was performed by the authors, first separately, then together to reach consensus and validate the findings.

Key results: Wheelchair users have some positive experiences with activity tracking using GPS-data e.g. some tracked whether the relationship between distance and time improved. However, they experienced that distance and time are the only parameters that are correctly measured, whereas calorie consumption, pulse, step and training intensity are not appropriately measured. The wheelchair users especially noted that some activity trackers provide reminders that assume that the user can walk, such as "it is time to get up and move around", which they considered meaningless. The wheelchair users experienced an un-met need to evaluate whether their overall physical activity was sufficient or whether they were too inactive, but none of the technologies that they had utilized, could provide this information, which includes exercise training as well as daily household tasks. In addition, the wheelchair users suggested that it would be motivating to compare current and previous achievements and compete with oneself and others. A surprising finding 
was that wheelchair users experienced a need of understanding their own typical pulse alterations, to be able to prevent dizziness related to performance of physical activity. In addition, the wheelchair users were concerned that physical activity measures could be used against them and become a barrier for granting assistive technology.

Conclusion: Sound activity trackers are needed, specifically designed for wheelchair users. The benefits hereof are evident from a public health perspective, because we might identify wheelchair users with low physical activity levels. In an assistive technology perspective, appropriate use of information provided by activity trackers should be considered to avoid distrust among wheelchair users. In summary, activity tracking may be valuable for wheelchair users, health care and society at large. Future research should account for the limitations of this study i.e. few participants limited to basketball players.

Keywords: activity tracking, wheelchair users.

*Corresponding author. E-mail: tih@ucn.dk

\section{Addressing Communication Issue Among Care- givers and Wheelchair Users: Identifying Design Metrics and Defining Needs}

Santosh Maurya ${ }^{\mathrm{a}, *}$, Daisuke Matsuura ${ }^{\mathrm{a}}$, Takahiro Uehara $^{\mathrm{b}}$, Masato Kawabata ${ }^{\mathrm{b}}$ and Yukio Takeda ${ }^{\mathrm{a}}$

${ }^{a}$ Department of Mechanical Engineering, Tokyo Institute of Technology, Tokyo, Japan

${ }^{\mathrm{b}}$ Hirakata General Hospital for Developmental Disorders, Osaka, Japan

Background: Needs for designing assistive technology devices (ATDs) are directly affected by variations in assistance required by the ATDs user, but people around ATDs user also affect definition of design needs as they interact with users on daily basis. Thus, other than functional aspect of ATDs, social and psychological aspects also gain importance. Here, we address the issue of difficulties in face to face communication during the process of assistance, among caregivers (CG) and wheelchair users (WU). Previous studies addressing this issue present new technical tools like autonomous mobility systems or novel wheelchair controllers. However early on in the design phase, it is difficult to assess nature of design problem required to be solved - whether in form of an original design or as new addons. In this work we narrow down the key metrics within the requirement-space that establish good correlations, focussing on improved communication experience and reduced stress for CGs.
Method: We conducted an exploratory study with a rehabilitation centre, and collected data in form of interviews and questionnaires with WU and CG (hospital staff and family members) as target participants. We designed a questionnaire targeting WU's activities, CG's workload, type of ATDs used, device handling problems, communication methods used and communication limitations. The collected data was analysed to narrow down relations among defined metrics statistically and qualitatively. The first phase of data collection and analysis lasted 1 month, establishing exhaustiveness of the designed questionnaire. 5 CG and 7 WU $(5 \mathrm{M}, 7 \mathrm{~F})$ with mean age of 45.6 years $(\mathrm{SD}=10.2)$ with mean experience of caregiving/receiving 4.58 ( $\mathrm{SD}=$ 1.16 , on a scale of $0-5)$, participated at first phase. CGs reported to provide assistance to $15.08(\mathrm{SD}=10.99)$ wheelchair users daily, approximately 8.9 times $(\mathrm{SD}=$ 4.31) per day.

Key results: Significant correlations among CG's $d u$ ration to get tired and frequency of assistance being provided were observed, e.g. +0.967 for night time all week, +0.893 and +0.898 for weekday and weekend afternoon time. Using factor analysis (FA) we grouped WU' activity and established differences in three components: personal-amusement (watching TV/reading, time with family), task-activities (going to shopping/hospital, playing indoors) and dailynecessities (toothbrushing, changing clothes). Also, using FA we narrowed down device handling problems to two components: mediated-non-mediated problem (passing through gates, static to dynamic) and deviceusability problems (power needed, ease of rotation). Results pointed out communications among WU and CG happened mostly at start of the assistance activity ( 40\%), where CG were dominant through speaking and touch. CG faced moderate difficulty with WU's voice and head-movement, and high difficulty with CG's bending their body for communication.

Conclusion: The analytical study helps to narrow down needs in form of correlated design metrics for WU-CG communication, and targets enhancing the communication experience. This serves as the first step to establish novel concept designs to intangible problems related to ATDs like wheelchair. The sample size needs to be expanded further to generalise the findings. The future prospect for this work is to generate and analyse design concepts, and evaluate them based on the established co-related design metrics.

Keywords: communication, caregivers, mobility, wheelchair users, design metrics.

${ }^{*}$ Corresponding author. E-mail: takeda@mech.titech. ac.jp 
Usability Assessment of a Navigation Tool for Manual Wheelchair Users in Urban Areas

François Routhier ${ }^{\mathrm{a}, \mathrm{b}, *}$, Camillle Lainesse-Morin ${ }^{\mathrm{a}, \mathrm{b}}$, Mir Abolfazl Mostafavi ${ }^{\mathrm{a}, \mathrm{c}}$ and Luc Noreau ${ }^{\mathrm{a}, \mathrm{b}}$

${ }^{a}$ Centre for interdisciplinary research in rehabilitation and social integration, Centre intégré de santé et de services sociaux de la Capitale-Nationale, 525 Hamel est, Québec, G1M 2S8, Canada

${ }^{\mathrm{b}}$ Department of rehabilitation, Université Laval, Québec, G1V 0A6, Canada

${ }^{\mathrm{c}}$ Department of geomatics sciences, Université Laval, Québec, G1V 0A6, Canada

Background: Manual wheelchair (MWC) users have many challenges to overcome when traveling outdoors (slopes, thresholds, holes, sleepers, road works, etc.). They must experiment different paths to find the one that suits them best. Currently navigation tools are not necessarily adapted to the needs of MWC users. These technologies provide some information on the accessibility of service points such as stores, restaurants and etc. but not the accessibility of the road network. The development of an innovative navigation technology called MobiliSIG (mobilisig.scg.ulaval.ca), based on the wheelchair use confidence, made it possible to fill this gap by proposing a mobile application that can be used on different types of smart devices. The objective of this study is to assess the usability of MobiliSIG with potential MWC users. Usability is the degree to which a technology can be used to achieve specific objectives with effectiveness, efficiency, and satisfaction. Method: Three men and three women (age: 2652 years; MWC experience: $5-36$ years; MWC skills: 22-91\%) participated in an experiment on two distinct routes in a district of Quebec City (Canada). They were interviewed to evaluate MobiliSIG according to 25 usability criteria. They had to express their responses using a scale between 1 representing 'not usable at all' and 5 'strongly usable'. Consistency between the level of the accessibility of each segment proposed by MobiliSIG and the objective evaluation of the participants (using a scale between 1-5 [1 representing 'strongly disagree' and 5 'strongly agreeing']) was also assessed. A quantitative and qualitative analysis of the data highlighted the advantages, disadvantages of the functionalities of MobiliSIG and several improvements were proposed.

Key results: The overall average of the ratings obtained for the usability criteria is high $(4.1 \pm 0.6 / 5)$. The top five criteria are: everyday vocabulary, ease of learning, endurance, assistance and support, and time spent for waiting. The five lowest rated criteria are: appearance, evidence of control, expected functionality, efficiency and effectiveness. The level of the accessibility of the segments according to MobiliSIG and that perceived by the participants are coherent $57 \%$ of the time (41 on 72 analysed segments of routes). For other segments, the level of perceived difficulty was higher for 20 segments and easier for 11 segments.

Conclusion: MobiliSIG is a promising tool for MWC users who want navigating in the City. This first iteration of MobiliSIG's usability assessment was generally positive. However, few points that will guide the future developments of this navigation tool were identified. Many technological challenges remain (e.g. improved positioning of the user, automation of the accessibility information extraction, considering changes in the environment and improved assessment of the accessibility) and should be considered for future development. As other perspectives, MobiliSIG should also be developed to include other profiles than MWC users, for instance, individuals who use other mobility device, blinded persons, etc. It should also integrate other modes of transportation for a complete trajectory, such as public transportation (e.g. bus and tramway).

Keywords: manual wheelchair, navigation, usability, effectiveness, efficiency, satisfaction.

*Corresponding author. E-mail: Francois.Routhier@ rea.ulaval.ca 